\title{
Step and kink correlations on vicinal Ge(100) surfaces investigated by electron diffraction
}

\author{
C. Tegenkamp, J. Wollschläger, H. Pfnür,* F.-J. Meyer zu Heringdorf, ${ }^{\dagger}$ and M. Horn-von Hoegen \\ Institut für Festkörperphysik, Universität Hannover, Appelstraße 2, D-30167 Hannover, Germany \\ (Received 15 February 2002; revised manuscript received 27 March 2002; published 29 May 2002)
}

\begin{abstract}
Using spot profile analysis in low-energy electron diffraction, we have investigated vicinal Ge(100) surfaces, which were miscut by $2.7^{\circ}$ and $5.4^{\circ}$, respectively, in [011] direction with respect to the surface normal. Within the kinematic approximation the morphology was evaluated quantitatively both perpendicular and parallel to the step edge direction. In contrast to vicinal $\mathrm{Si}(100)$ surfaces with similar miscut angles, the $\mathrm{Ge}(100)$ surfaces still show an alternating configuration of $(2 \times 1)$ and $(1 \times 2)$ reconstructed $(100)$ terraces, which are separated by steps of single atomic height. From the spot profiles and their energy dependence we extracted the morphological parameters such as the average terrace width, the variance of the terrace size distribution, and the average kink separation. Furthermore, step energies on the vicinal $\mathrm{Ge}(100)$ surfaces were estimated. These turn out to be significantly lower than for $\mathrm{Si}(100)$ and lead to the formation of the observed double domain structure.
\end{abstract}

DOI: $10.1103 /$ PhysRevB.65.235316

PACS number(s): 61.14.Hg, 61.72.Lk, 68.35.Bs, 68.35.Md

\section{INTRODUCTION}

Investigations of the morphology of surfaces (especially of semiconductors) have become of great interest in the last years. Especially the equilibrium structures of vicinal surfaces have been studied in detail both theoretically and experimentally, ${ }^{1-3}$ since these surfaces are easy to produce and are well ordered. They also bear some potential for manufacturing periodically arranged extremely small structures, e.g., by taking advantage of the preferential adsorption of atoms at steps.

Compared to $\mathrm{Si}$, a very small number of studies have been carried out on vicinal Ge surfaces concerning the preparation of different morphologies as caused, e.g., by the competition between the formation of step bunches separated by large terraces and small terraces separated by single atomic steps. Predominantely for $\mathrm{Si}(111)$ surfaces the effect of step-step interaction has been studied extensively. ${ }^{4}$ In analogy to $\mathrm{Si}(111)$ also $\mathrm{Si}(100)$ surfaces with miscut in the [011] direction undergo a transition from steps of single to double atomic height with increasing miscut angle. ${ }^{5}$ This effect is caused by the competition between both the stress from the terrace due to the reconstruction and the stress at step sites.

To describe quantitatively the surface morphology of vicinal Si surfaces, the concept of surface stress ${ }^{6}$ has been applied successfully in the past. ${ }^{7-9} \mathrm{Ge}(100)$ surfaces are very similar to $\mathrm{Si}(100)$ surfaces, they show $(1 \times 2)$ and $(2 \times 1)$ reconstructions, e.g., but the step structure on vicinal surfaces seems to be different. Therefore, we studied the morphology of these surfaces by carrying out highly resolved low-energy electron diffraction (LEED) experiments on $\mathrm{Ge}(100)$ surfaces misoriented by $2.7^{\circ}$ and $5.4^{\circ}$ with respect to the surface normal.

There is also a renewed interest in the geometrical structure of Ge surfaces, not only to prove the physical concepts developed for vicinal Si surfaces. Devices based on $\mathrm{Si} / \mathrm{Ge}$ heterostructures are also technologically important. Our interest in the morphology of Ge surfaces is coupled with studies of epitaxy of wide band gap insulating films on Ge surfaces (e.g., $\mathrm{NaCl}$ or $\mathrm{KCl}$ ) as model insulator/semiconductor systems. The simplest way to structure an insulating film is by structuring the substrate surface, if epitaxial growth is still possible. Since the smallest step height in $\mathrm{Ge}$ is half as high as for $\mathrm{NaCl}, \mathrm{Ge}$ steps of single atomic height are overgrown by $\mathrm{NaCl}$ mosaics. ${ }^{10}$ Therefore, doubly stepped $\mathrm{Ge}(100)$ surfaces are needed. We have recently shown that steps in $\mathrm{NaCl}$ films can indeed be induced by doubly stepped vicinal $\mathrm{Ge}(100)$ substrates. ${ }^{11}$

Our experiments complement investigations carried out with scanning tunneling microscopy (STM), ${ }^{12}$ in the manner that our investigations are for higher miscut angles than those investigated by STM. We also demonstrate that LEED experiments combined with spot profile analysis (SPALEED) are well-suited to obtain independent information about both the terrace size distributions and the step roughness, i.e., the kink separation and correlation length. These experiments have the advantage to give automatically a precise average value of the quantities investigated.

\section{EXPERIMENT}

The experiments have been carried out in a UHV chamber, equipped with SPA-LEED, ultraviolet photoemission spectroscopy (UPS), x-ray photoemission spectroscopy (XPS), and a mass spectrometer, at a base pressure of 1 $\times 10^{-8} \mathrm{~Pa}$. The vicinal Ge(100) crystals (Crystec, Berlin) have been polished mechanically by diamond paste followed by a final chemical treatment. The precision in the polar direction was specified to be better than $0.2^{\circ}$. Azimuthal orientation was better than $\pm 2^{\circ}$ as shown with $\mathrm{x}$-ray diffraction. Samples with $2.7^{\circ}$ and $5.4^{\circ}$ off the [001] direction towards [011] have been prepared. (Nomenclature in the following will be $\mathrm{Ge}(100)-[011] 2.7^{\circ}$ and $\mathrm{Ge}(100)-[011] 5.4^{\circ}$.) These crystals have been mounted on a transferable sample holder, which allowed heating by both direct current and by radiation or electron bombardment through a filament located behind the sample surface, respectively. The temperature was controlled by a Ni/Ni-Cr thermocouple attached to the sample holder.

Before transfering the crystal into the UHV chamber we 
carried out an ozone treatment to reduce the carbon contamination. ${ }^{13}$ The cleanness of the surface was controlled by XPS and x-ray induced Auger electron spectroscopy (XAES). This treatment reduced the amount of carbon significantly, as checked by XPS, but was not able to completely remove all carbon on the stepped surfaces. Therefore, the vicinal $\mathrm{Ge}(100)$ crystals have been cleaned additionally. After removal of the oxide, by annealing the crystal for at least $2 \mathrm{~h}$ at $600 \mathrm{~K}$, cycles of bombardment with $800 \mathrm{eV} \mathrm{Ar}^{+}$ ions (crystal current typically $1.5 \mu \mathrm{A}$ ) followed by annealing to $900 \mathrm{~K}$ for at least 2 min were carried out. All SPA-LEED investigations (transfer width of $1000 \AA$ ) were done afterwards at room temperature.

\section{RESULTS}

In this section we first present qualitative results of vicinal Ge surfaces as revealed by our LEED study. Thereafter, in Sec. III A, terrace size distributions are determined by analyzing spot profiles normal to the step direction. Information about the average step roughness was obtained from profiles of the diffuse intensities between split spots in the direction parallel to the steps, as shown in Sec. III B.

An overview LEED pattern of a freshly prepared vicinal $\mathrm{Ge}(100)$-[011] $5.4^{\circ}$ surface at an anti-Bragg (out-of-phase) condition $^{14}$ with regard to the $(00)$ spot is shown in Fig. 1. It is a great advantage of LEED that directly from such a snapshot important details for a surface model can be concluded.

First, it shows the $(2 \times 1)$ reconstruction with two rotational domains that is characteristic of the Ge(100) surface. Due to the $\sigma$ bonding between two surface atoms and the atomic relaxations, the $(1 \times 1)$ surface transforms to a $(2 \times 1)$ or $(1 \times 2)$ superstructure where the orientation of the domains depends on the terrace according to the symmetry of the diamond lattice of Ge. ${ }^{15}$ The miscut leads to the characteristic splitting of all integer spots, whereas the half order spots are only broadened. The splitting of the fundamental spots indicates the existence of a sequence of steps in [011] direction. Therefore, the splitting of $26 \%$ SBZ (surface Brillouin zone) corresponds to an average terrace length of $15 \AA$ assuming steps with single atomic height, in agreement with the miscut angle. Interestingly, despite the high miscut angle of $5.4^{\circ}$, both rotational domains are still visible. This means that the vicinal $\mathrm{Ge}(100)$ surfaces consist of terraces that are (at least partly) separated by steps of single atomic height. Similar results are obtained for the $\mathrm{Ge}(100)-[011] 2.7^{\circ}$ surface (not shown).

Second, it is characteristic of these surfaces (see Fig. 1) that the spots of the $(2 \times 1)$ reconstruction are more intense than the diffraction spots of the $(1 \times 2)$ domain. Since this was found at many different scattering conditions (i.e., electron energies), the average terrace length of the $(2 \times 1)$ domain must be larger than that of the $(1 \times 2)$ domain, as shown schematically in Fig. 2. This result is in agreement with the nonvanishing central spot at the anti-Bragg condition. Due to the preferential termination of the surface by one type of terrace, the extinction at an out-of-phase condition with respect to adjacent terraces cannot be complete. Although this argument is strictly valid only for the case that the incoming

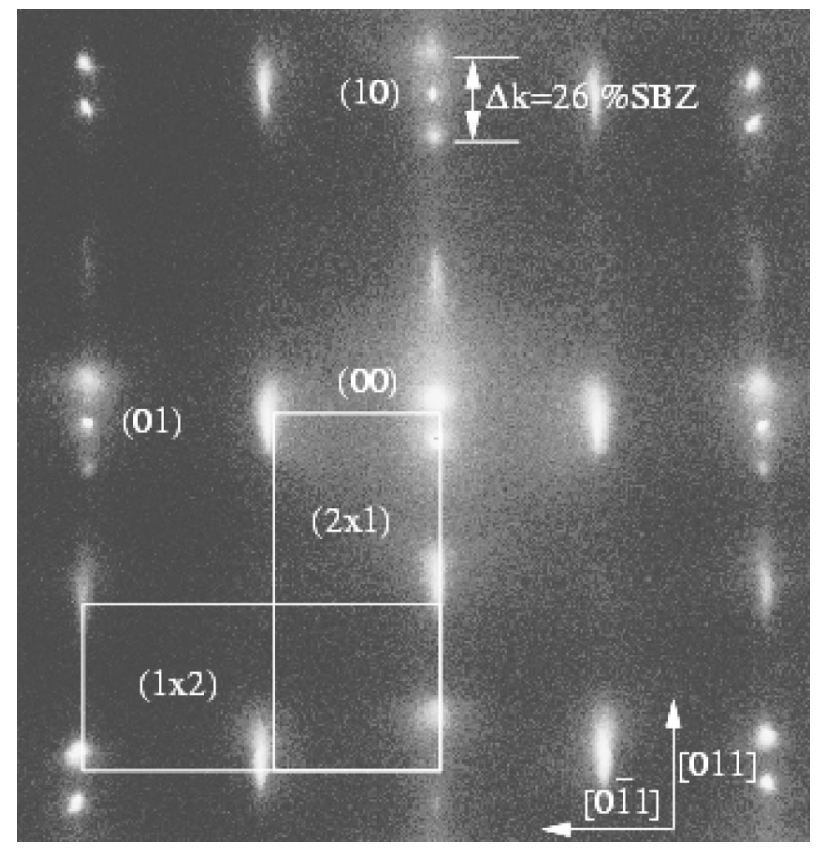

FIG. 1. LEED pattern of the first SBZ of a Ge(100) - [011]5.4 ${ }^{\circ}$ surface at an electron energy of $230 \mathrm{eV}$. Besides the spot splitting of the integer spots, also both $(2 \times 1)$ reconstruction domains are visible, indicating the existence of steps of single atomic height. $T=300 \mathrm{~K}$.

and the scattered wave vectors are perpendicular to the surface to avoid any dynamical effects, it should be still correct at the small scattering angle used in SPA-LEED $\left(\approx 7^{\circ}\right)$. Thus, the LEED results show that the Ge(100) surfaces have mainly single atomic steps. For purely doubly stepped surfaces as for $\mathrm{Si}(100)$ with similar miscut, ${ }^{16}$ one reconstruction domain vanishes. These qualitative results do not exclude the existence of a small fraction of steps with double atomic height. Extrapolating from previous STM measurements ${ }^{12,17}$ to the $\mathrm{Ge}(100)-[011] 2.7^{\circ}$ surface, there should be a fraction of double steps of less than $10 \%$, but it could be significantly higher for the $\mathrm{Ge}(100)-[011] 5.4^{\circ}$ surface.

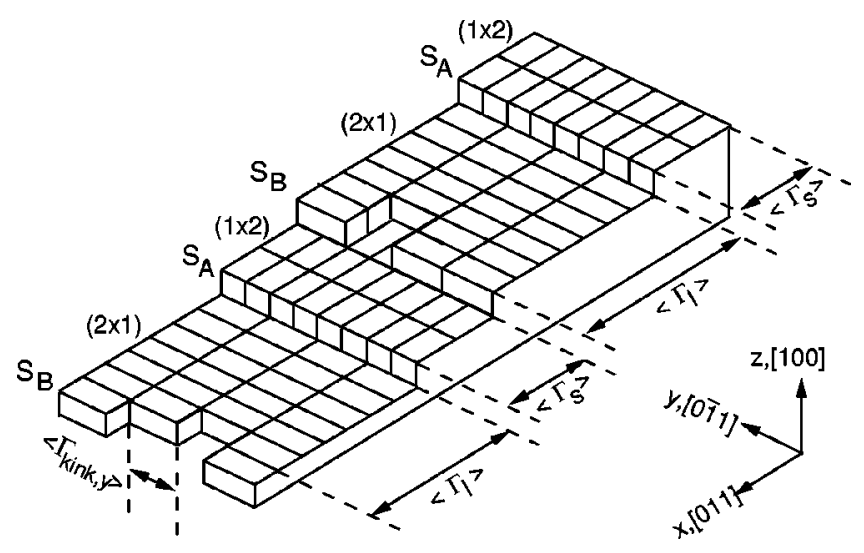

FIG. 2. Schematic view of a $(1 \times 2)$ reconstructed (100) surface tilted towards the [011] direction with rough $S_{B}$ steps and smooth $S_{A}$ steps. The geometrical parameters are explained in the text. 
In case of vicinal surfaces, correlations are directly observable with LEED. Assuming identical scattering amplitudes for both steps and terraces, the splitting of the integer spots vanishes at any in-phase condition, since the spots due to the periodic array of steps are located at $2 \pi /\langle\Gamma\rangle$, which are also the positions of the minima of an envelope function originating from the finite terrace length. ${ }^{18}$ Therefore, only for non-in-phase scattering conditions the spot splitting is visible. The degree of correlation between steps is considerably less for vicinal $\mathrm{Ge}(100)$ compared to stepped $\mathrm{Si}(100)$, as judged from the number of spots visible for a non-in-phase condition. Whereas for vicinal Ge(100) surfaces a maximum of three spots are visible (cf. Fig. 1), for corresponding $\mathrm{Si}(100)$ surfaces up to five satellites have been observed, ${ }^{16}$ even at Bragg point positions. An alternative explanation for this difference could be that scattering amplitudes are more or less the same for terrace and step atoms in the case of $\mathrm{Ge}(100)$ but not for vicinal $\mathrm{Si}(100)$. This, however, does not seem to be the main reason for this difference, as discussed below.

\section{A. Terrace size distribution (step-step distance)}

In order to obtain deeper insight into the morphology of the vicinal Ge surfaces, we have measured one-dimensional LEED spot profiles along the [011] direction for various electron energies. These are transformed into plots of $k_{\perp}$ versus $k_{\|}$using a gray scale representation for the intensity. Such a plot corresponds to a two-dimensional vertical cut through the reciprocal space at the azimuth given by $k_{\|}$and is shown in Fig. 3. The tilted rods with the same inclination are characteristic for regularly stepped surfaces (i.e., for surfaces with a narrow distribution of step distances). ${ }^{16}$ Also visible is a broad rod at $50 \% \mathrm{SBZ}$ that does not vary in $k_{\|}$. It is due to a superstructure spot from the $(1 \times 2)$ minority domain.

As seen qualitatively from this figure, the full width at half maximum (FWHM) of these LEED spots varies as a function of $k_{\perp}$ with pronounced minima at the threedimensional Bragg positions. By fitting the one-dimensional line scans with Lorentzian functions, we have determined the FWHMs of the (00) spot. In Fig. 4, we plotted them versus the scattering phase $S$ for both the $\mathrm{Ge}(100)-[011] 2.7^{\circ}$ and the $\mathrm{Ge}(100)-[011] 5.4^{\circ}$ surface. As seen from this figure, the functional dependence can be described by parabolas with minima at the Bragg positions. This behavior seems to be characteristic for vicinal Ge(100) surfaces.

This functional form can be explained using a model that is based on the qualitative findings discussed in context of Figs. 1 and 2, i.e., on small correlations between terraces. In our model we assume that only steps of monatomic height exist. The only correlation assumed was the alternating sequence of short $(1 \times 2)$ and long $(2 \times 1)$ domains. Long and short terraces are characterized by individual terrace length distributions, i.e., by their average terrace lengths, $\left\langle\Gamma_{l}\right\rangle$ and $\left\langle\Gamma_{s}\right\rangle$, respectively, and by their variances $\sigma_{l}$ and $\sigma_{s}$. Within these restrictions and with the average step density $1 /\langle\Gamma\rangle$, fixed by the miscut angle, the terrace length was chosen randomly (Markovian chain). Details of the calculation will be

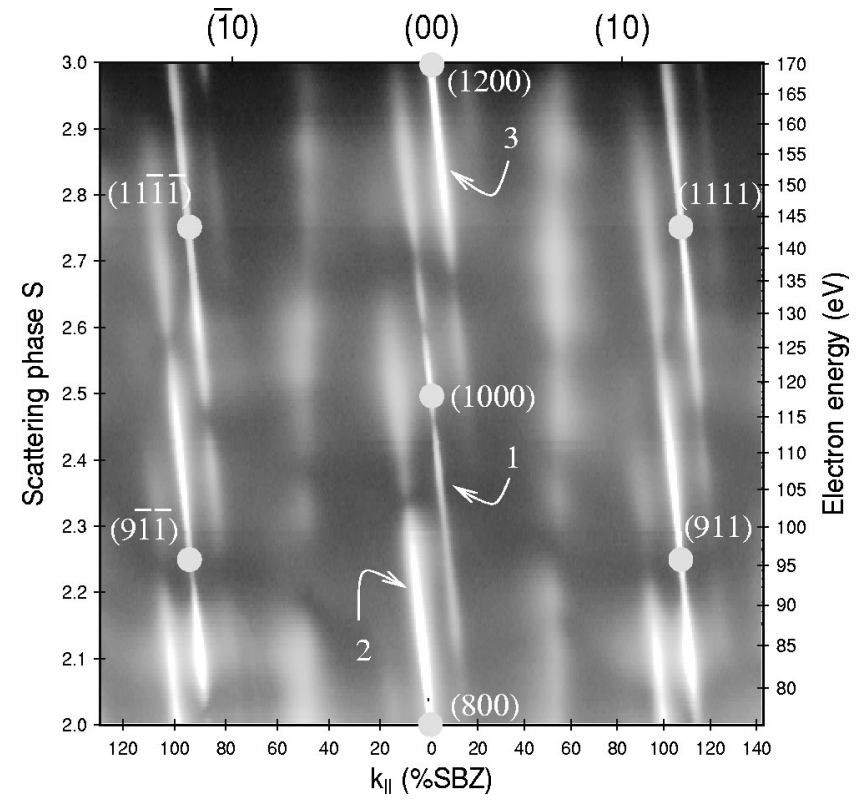

FIG. 3. $\left(k_{\|}, k_{\perp}\right)$ scan along the [011] direction of the first Brillouin zone for the $\mathrm{Ge}(100)-[011] 5.4^{\circ}$ surface. The closed circles indicate the Bragg points of the diamond lattice and the labels " 1 ," " 2 ," and "3" belong to the parabolas shown in Fig. 4.

published elsewhere ${ }^{19}$ Here we only discuss the results. For the case of sharp terrace size distributions $\left(\sigma_{l} \ll\left\langle\Gamma_{l}\right\rangle\right.$ and $\left.\sigma_{s} \ll\left\langle\Gamma_{s}\right\rangle\right)$ our model predicts that the diffraction spot profiles of the tilted rods broaden systematically, if the scattering condition deviates from $S=n$ or $S=n+\frac{1}{2}$. From a detailed analysis we obtain for the normalized FWHM $\left(F_{\text {norm }}\right)$ with respect to the SBZ the parabolic behavior

$$
F_{n o r m}=8 \pi \frac{a \sigma^{2}}{\langle\Gamma\rangle^{3}}(\delta S)^{2},
$$

where $\delta S$ denotes the deviation of the scattering phase $S$ $=n$ or $S=n+\frac{1}{2}$. This is exactly the experimentally observed parabolic behavior of the half-widths. Equation (1) can only be used to calculate the combined variance $\sigma^{2}:=\sigma_{l}^{2}+\sigma_{s}^{2}$ by

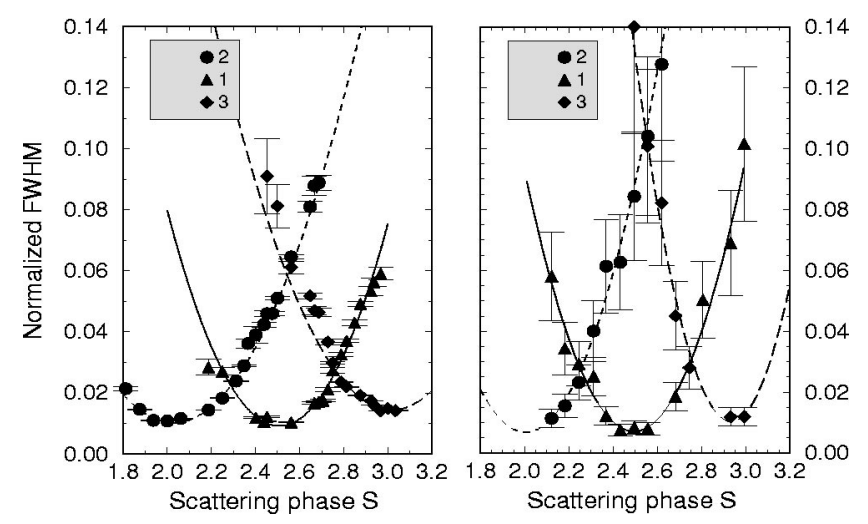

FIG. 4. Normalized FWHM, $F_{\text {norm }}$, in the $x$ direction of the rods, labeled 1, 2, and 3 in Fig. 3, versus the scattering phase $S$ for the $\mathrm{Ge}(100)-[011] 2.7^{\circ}$ (left) and $\mathrm{Ge}(100)-[011] 5.4^{\circ}$ (right) surface. The lines are obtained from parabolic fits according to Eq. (1). 
fitting this function to the experimental data (see lines in Fig. 4). For the ratio $\sigma /\langle\Gamma\rangle$ we obtain values of 0.33 and 0.32 for the $\mathrm{Ge}(100)-[011] 2.7^{\circ}$ and the $\mathrm{Ge}(100)-[011] 5.4^{\circ}$ surfaces, respectively. Therefore, we can justify the assumption of narrow terrace size distributions a posteriori, and show that the model is consistent with the experimental data.

However, the curvature of the parabolas is only implicitly determined by $\left\langle\Gamma_{l}\right\rangle,\left\langle\Gamma_{s}\right\rangle, \sigma_{l}$, and $\sigma_{s}$. An independant measurement of $\left\langle\Gamma_{l}\right\rangle$ and $\left\langle\Gamma_{s}\right\rangle$ can be obtained by a $G(\Theta)$ analysis. The two distributions of short and long terrace sizes lead to an unequal occupation probability of surface atoms on these terraces. Therefore, the intensity of the central spot at the out-of-phase condition with respect to monatomic steps ( $S=n+\frac{1}{2}$, e.g., $(10,0,0)$ in Fig. 3$)$ is nonzero. Within our model the normalized intensity for such a condition is given by $^{20}$

$$
G_{0}\left(p, k_{\perp}^{\text {out }}\right):=\frac{I\left(k_{\|}=0, k_{\perp}^{\text {out }}\right)}{I_{\text {total }}}=p^{2}
$$

where $I_{\text {total }}$ denotes the integrated intensity of the (00) spot (central spot and satellites due to the adjacent tilted rods). $p$ is the asymmetry parameter defined by $\left\langle\Gamma_{i}\right\rangle=(1 \pm p)\langle\Gamma\rangle$. The positive sign refers to the long islands. The average terrace length, $\langle\Gamma\rangle$, is given by $\langle\Gamma\rangle=\left(\left\langle\Gamma_{s}\right\rangle+\left\langle\Gamma_{l}\right\rangle\right) / 2$. In this context $p=1$ denotes the $(2 \times 1)$ single domain structure with steps of double atomic height.

In order to extract the geometrical parameters $\left\langle\Gamma_{l}\right\rangle,\left\langle\Gamma_{s}\right\rangle$, $\sigma_{l}$, and $\sigma_{s}$ for both types of terraces individually, we assume scaling for the ratios of variances and terrace lengths, i.e., $\sigma_{s} /\left\langle\Gamma_{s}\right\rangle=\sigma_{l} /\left\langle\Gamma_{l}\right\rangle$. This assumption was found to be fulfilled for vicinal $\mathrm{Si}(111)$ surfaces, as determined by STM. ${ }^{21}$ Our results for both the $\mathrm{Ge}(100)-[011] 2.7^{\circ}$ and the $\mathrm{Ge}(100)-[011] 5.4^{\circ}$ surfaces are shown in Fig. 5, where we also compare them with those obtained earlier by $\mathrm{STM}^{17}$ on surfaces with $1^{\circ}$ and $2^{\circ}$ miscuts. In units of next-neighbor distances we obtained for the $\mathrm{Ge}(100)-[011] 2.7^{\circ}$ surface $\left\langle\Gamma_{l}\right\rangle=9.1,\left\langle\Gamma_{s}\right\rangle=5.9, \sigma_{l}=2.9, \sigma_{s}=1.9$, and for the $\mathrm{Ge}(100)-[011] 5.4^{\circ}$ surface $\left\langle\Gamma_{l}\right\rangle=5.5,\left\langle\Gamma_{s}\right\rangle=1.9, \sigma_{l}=2.7$, $\sigma_{s}=0.9$. As seen from Fig. 5, our data nicely extend the trends found with STM for the $1^{\circ}$ and $2^{\circ}$ misoriented $\mathrm{Ge}(100)$ surfaces to higher step densities. These results demonstrate directly the complementarity of STM and LEED. In addition, we plotted the asymmetry factor $p$ in the right part of Fig. 5. Even for the Ge(100)-[011]5.4 ${ }^{\circ}$ surface $p$ is far away from unity, i.e., even the surface with the highest step density investigated here is far from a surface containing mainly double steps. A linear extrapolation leads to a phase transition angle of approximately $9^{\circ}$, which is three times higher than for $\mathrm{Si}(100)$.

Furthermore, the model of the alternating arrangement of long and short terraces allows inherently the calculation of the density of steps with double atomic height. The $D_{B}$-step density is given by the probability of finding $S_{A}$ steps with a terrace length of $1.5 a$. Assuming a Gaussian distribution we obtain for the $\mathrm{Ge}(100)-[011] 5.4^{\circ}$ surface a $D_{B}$-step density of approximately $50 \%$, which is in reasonable agreement
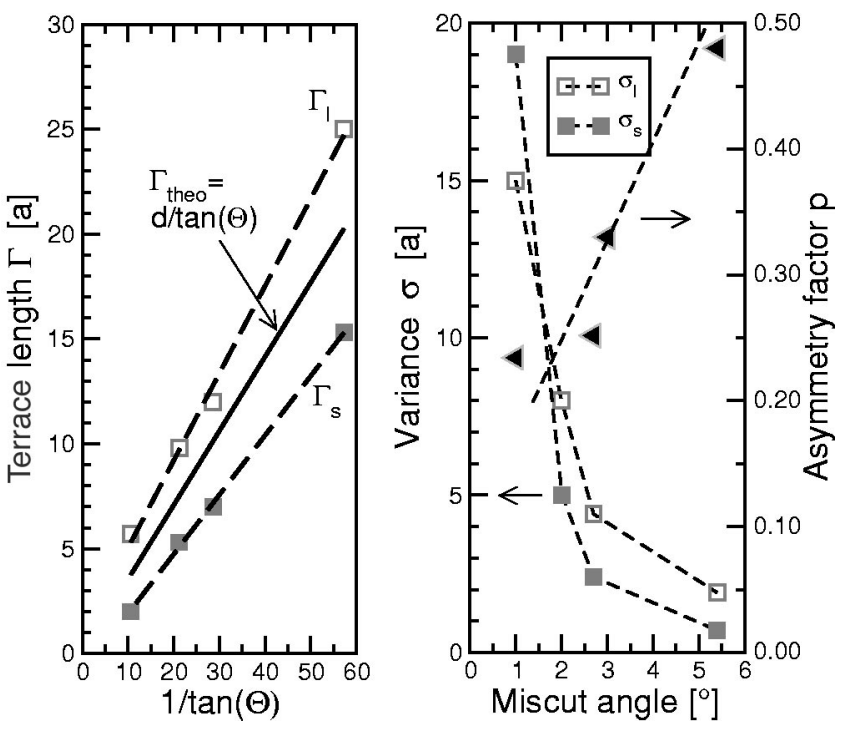

FIG. 5. Results of the terrace lengths (top) and the variances (bottom) for the long [subscript $l,(2 \times 1)$ reconstructed] and the short [subscript $s,(1 \times 2)$ reconstructed] terraces. Data for the miscuts of $2.7^{\circ}$ and $5.4^{\circ}$ are from this work, obtained with LEED. Results for the $1^{\circ}$ and $2^{\circ}$ misoriented surfaces are from Ref. 17 and were obtained with STM.

with STM results. ${ }^{17}$ On the contrary, the step density with double atomic height for the $\mathrm{Ge}(100)-[011] 2.7^{\circ}$ surface is less than $1 \%$.

\section{B. Step roughness}

LEED provides the full two-dimensional information in $k$ space. In the following we will use this information contained in profiles in the direction along the step edges to obtain values about the average step roughness.

In Fig. 6(a) we have plotted the (00) spot of the $\mathrm{Ge}(100)-[011] 2.7^{\circ}$ surface near an anti-Bragg condition. The three bright spots seen are characteristic for the vicinal surface. They correspond to the intersection with the rods shown in Fig. 3. Here we want to concentrate on the distribution of diffuse intensity in both $k_{x}$ and $k_{y}$ directions. As seen, there is considerable diffuse intensity between the rods, which is spread out more and more in $k_{y}$ direction for larger $k_{x}$ values. For perfectly smooth and strictly periodic step distances this diffuse intensity should be completely absent. However, for a variation of step distances between straight steps, the diffuse intensity should only extend in $k_{x}$ direction. It is the meandering of steps that causes the diffuse intensity to be spread out perpendicular to the direction of vicinality. Therefore, it contains directly information about the roughness. The comparatively sharp concentration of this diffuse intensity around $k_{y}=0$ and its quick broadening as a function of $k_{x}$ is an indication that the steps are rough with a correlation length $\xi$ along the steps larger than the kink-kink distance $\left\langle\Gamma_{\text {kink }, y}\right\rangle$.

In order to obtain more quantitative information about the average step roughness we assume that the step deviations from the average position of different steps are uncorrelated, and use a model of meandering, noncolliding steps. ${ }^{22}$ This 
a)

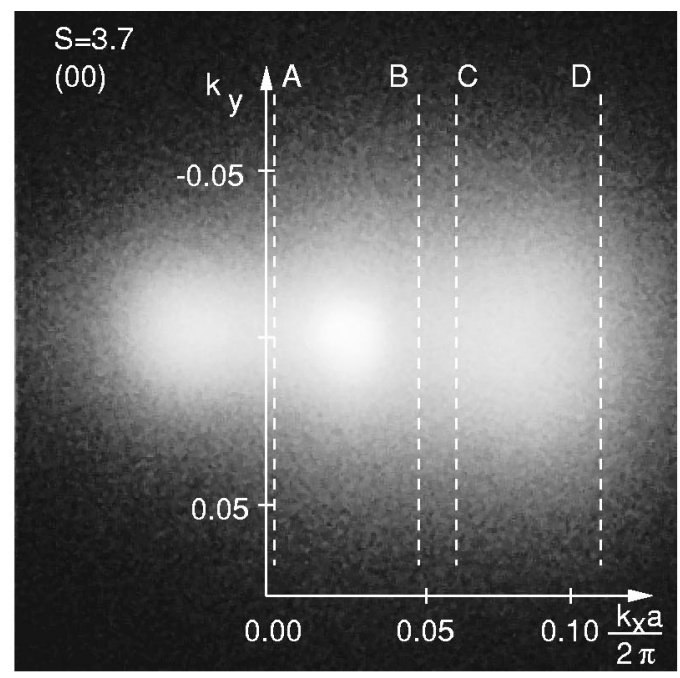

b)

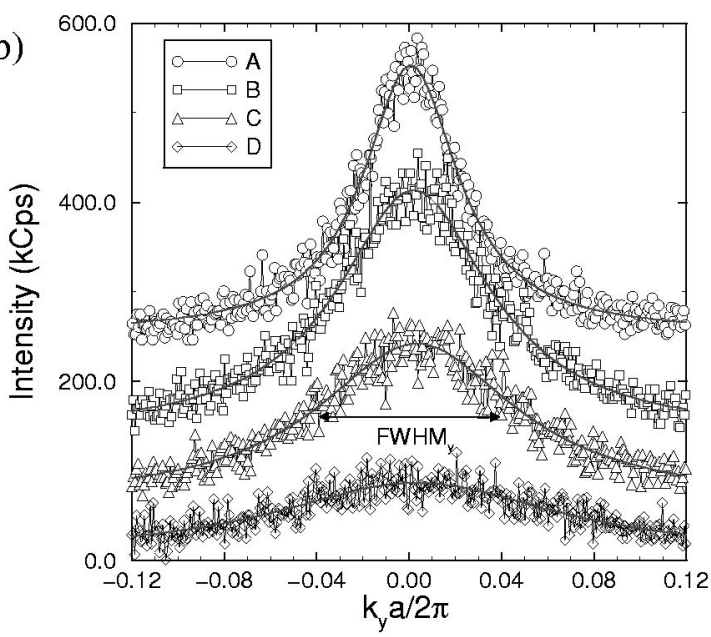

c)

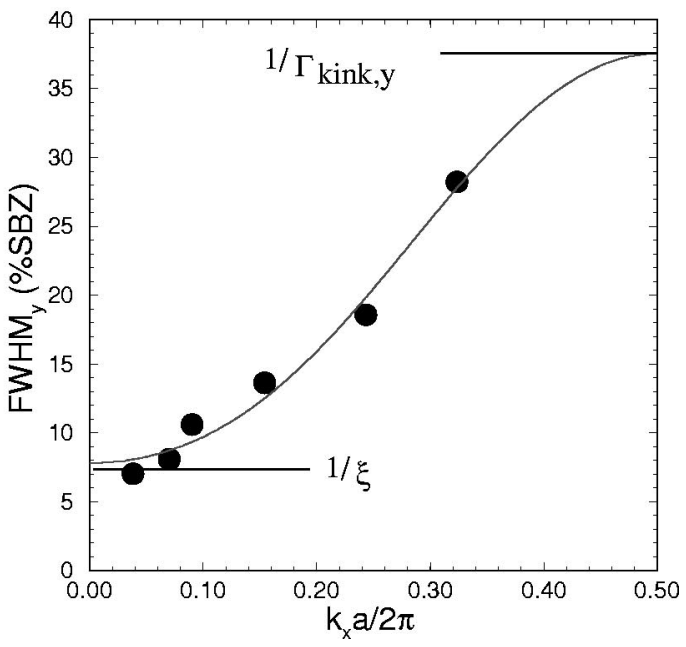

FIG. 6. (a) (00) spot of the $\mathrm{Ge}(100)-[011] 2.7^{\circ}$ surface near an out-of-phase condition. (b) Line scans in [011] direction for different $k_{x}$ values. The fit (solid lines) is done with lorentzian functions. For the better visibility the plots are shifted against each other in vertical direction. (c) The FWHMs, $F_{y}$, of (b) versus $k_{x}$. The solid line represents the fit according to Eq. (3). model does not distinguish between the two types of steps. Therefore, we obtain only an average information about the roughness. For exponentially decaying correlations of the fluctuations along a step, as assumed in this model, the information is contained in the half-widths of Lorentzian profiles of the diffuse intensity in $k_{y}$ direction as a function of $k_{x}$,

$$
F_{y} \approx \frac{2 w^{2}}{\xi a^{2}} \frac{1-\cos \left(k_{x} a\right)}{1-\left|\beta\left(k_{x}\right)\right|^{2}} .
$$

Here $w$ is the rms width of the step, $\xi$ is the correlation length along the steps, and $a$ denotes the lattice constant. The attenuation factor $\left|\beta\left(k_{x}\right)\right|^{2}=\exp \left\{-2\left(w^{2} / a^{2}\right)[1\right.$ $\left.\left.-\cos \left(k_{x} a\right)\right]\right\}$ is known as the static Debye-Waller factor. ${ }^{23}$ The evaluation of Eq. (3) should be done close to an antiBragg condition $\left(S=n+\frac{1}{2}\right)$. This equation shows that the correlation length $\xi$ can be determined from the limit $k_{x}$ $\rightarrow 0$. In this limit, $F_{y}$ (FWHM in the $y$ direction) goes to $1 / \xi$. The average terrace length can be extracted from the FWHM of LEED spots at an anti-Bragg condition. In principle, the average kink separation length $\left\langle\Gamma_{k i n k, y}\right\rangle$ is obtained at the boundary of the Brillouin zone $\left(k_{x} a=\pi\right)$ by the FWHM in the $y$ direction, but because of the low correlation there is practically no measurable intensity at $k_{x} a=\pi$. Therefore, $\left\langle\Gamma_{k i n k, y}\right\rangle$ can only be determined by extrapolation of the experimental data from lower values of $k_{x} a$ using Eq. (3). The rms width $w$ determines the curvature in Eq. (3). A detailed description of this model can be found in Ref. 22.

Following the model mentioned above, line scans along the [011] direction (parallel to the steps) were taken and fitted by Lorentzian functions, as shown in Fig. 6(b) for the sections marked $A$ to $D$ in Fig. 6(a) in between the diffraction spots. The half-widths as a function of $k_{x}$ are plotted in Fig. 6(c). From the fit (solid line) using Eq. (3) we obtained $\left\langle\Gamma_{\text {kink, } y}\right\rangle=2.6 a$ and for the correlation length $\xi=14.3 a$, respectively. The rms width was determined to be $w=1.6 a$.

It should be mentioned that, in general, the step creation energy for the $S_{A}$ step is significantly lower than for the $S_{B}$ step, e.g., more than one order of magnitude for the $\mathrm{Si}(100)$ surface. ${ }^{24}$ This leads to smooth $S_{A}$ and rough $S_{B}$ steps, which was also found for the Ge steps. ${ }^{17,25}$ Therefore, we have determined basically the roughness of the $S_{B}$-type steps from the SPA-LEED analysis. The statistical analysis done with STM reveals in case of $\mathrm{Si}(111)$ and $\mathrm{Si}(100)$ that the interaction between kinks can be almost neglected. ${ }^{26,27}$ As outlined in the preceding section, correlation effects and therefore interaction forces between Ge steps are much weaker than for Si steps, i.e., geometrical relaxations at step sites seem to be quite small. Thus also the interaction between kinks along a step is weak, i.e., the model of noninteracting kinks can be applied to our Ge data in accordance with previous STM measurements. ${ }^{28}$ According to Ref. 29, the diffusivity for an isolated step is uniquely determined by the kink energy, $\epsilon$, and by temperature, and can be expressed by $\langle k\rangle^{2}$ $\approx 2 e^{-\epsilon / k T} \cdot{ }^{30}$ Our crystal was heated to $900 \mathrm{~K}$ for several minutes before it was subsequently cooled slowly to room temperature, where the LEED experiment was done. Since 
the freeze-in temperature of the step edge roughness is around $400 \mathrm{~K},{ }^{28}$ the calculation of the diffusivity $\langle k\rangle^{2}$ for $\mathrm{Ge}(100)$ steps via $\langle k\rangle^{2}=2 w^{2} a \xi^{-1}$ (see Ref. 31) leads to a typical kink creation energy of approximately $70 \mathrm{meV} / 2 a$. Although we can only give an estimate of the kink creation energy because of the uncertainty of the freeze-in temperature, this estimate is in reasonable agreement with results from a detailed STM investigation done by Zandvliet. ${ }^{28}$ For the $S_{B}$-type step, a step free energy of approximately 80 $\mathrm{meV} / 2 a$ was obtained there. Although the uncertainty of our estimation is quite large, it still shows that the energetics of steps on vicinal $\mathrm{Ge}(100)$ differ significantly from corresponding $\mathrm{Si}(100)$ surfaces, as will be shown below.

\section{DISCUSSION}

In this paper we have presented results of the $\mathrm{Ge}(100)-[011] 2.7^{\circ}$ and $\mathrm{Ge}(100)-[011] 5.4^{\circ}$ surfaces investigated by SPA-LEED, and have quantified the fact that vicinal $\mathrm{Ge}(100)$ surfaces even at high miscut angles consist mainly of steps with single atomic height, in contrast to vicinal $\mathrm{Si}(100)$ surfaces that at these inclinations show single domain structures.

This difference between the vicinal (100) surfaces of Ge and $\mathrm{Si}$ needs some rational, which we outline below. It is based on our experimental data, together with reasonable estimates where experimental data are missing, and on the continuum mechanical model that has been used particularly for vicinal $\mathrm{Si}(100)$ surfaces by Alerhand et al. ${ }^{7}$ and later on by Pehlke et al. ${ }^{8}$ This model that considers only energetic, but no entropic contributions to the surface (free) energy, has been used to describe the phase transition from steps of monoatomic to double atomic height. Since Ge and Si have identical crystal lattices and also their (100) surfaces reconstruct in the same way, i.e., they form a dimer $(2 \times 1)$ reconstruction, an asymmetry with respect to the terrace size distribution is found also for $\mathrm{Ge}(100)$, except that the transition angle seems to be much higher than on $\mathrm{Si}(100)$. Within the theory just mentioned, the minimum in the surface energy is determined by both the stress anisotropy on the $(2 \times 1)$ and $(1 \times 2)$ reconstructed terraces and relaxation effects at step sites, which lead to additional stress contributions within the elastic theory. Following literature, the amplitudes of these two contributions are parametrized by $\lambda_{\sigma}$ and $\lambda_{d}$.

The interplay between these contributions and the different domain wall energies $\lambda_{S_{A}}$ and $\lambda_{S_{B}}$ for $S_{A^{-}}$and $S_{B^{-}}$-type steps of single atomic height and $\lambda_{D_{B}}$ for double steps, respectively, causes an asymmetry $p$ in the terrace size distribution. A detailed description of the individual energy terms can be found in Refs. 32 and 33. With increasing miscut angles, i.e., with the corresponding reduction of the average terrace lengths, the interaction between step sites is increased. Once a critical angle is obtained, the asymmetry becomes maximal, i.e., the surface forms a single domain structure with $D_{B}$-type steps.

In a simple model for the phase transition, where only the energy balance between vicinal surfaces consisting of steps with single and double atomic height ${ }^{3}$ is considered, the critical terrace length $\left\langle\Gamma_{c}\right\rangle$ is given by

$$
\left\langle\Gamma_{c}\right\rangle=\pi a \exp \left[\frac{\lambda_{S_{A}}+\lambda_{S_{B}}-\lambda_{D_{B}}}{2 \lambda_{\sigma}}\right] .
$$

We expect that the actual critical terrace lengths at finite temperatures are smaller than those calculated from this formula, since entropic contributions are completely neglected. However, for low temperatures and for a qualitative comparison intended here energetic considerations should be sufficient.

In our experiments we have determined the asymmetry parameter $p$, which for the minimum of the elastic energy is related to $\lambda_{\sigma}$ and $\lambda_{d}$ by (see Ref. 34) $p$ $=(1 / \pi) \sin ^{-1}\left[(a /\langle\Gamma\rangle) \sqrt{3 \lambda_{d} / \lambda_{\sigma}}\right] . p$ was determined to be 0.3 for the $\mathrm{Ge}(100)-[011] 2.7^{\circ}$ surface, i.e., the ratio of $\lambda_{d} / \lambda_{\sigma}$ is 10 .

The analysis of the LEED profiles has shown that the Ge(100)-[011]2.7 $7^{\circ}$ surface consists mainly of $S_{A^{-}}$and $S_{B}$-type steps, with a fraction of double steps lower than $1 \%$. If we assume the same domain wall energies as for $\mathrm{Si}(100)$, a $D_{B}$-step configuration results from Eq. (4) already for the $\mathrm{Ge}(100)-[011] 2.7^{\circ}$ surface, contrary to the experimental findings. Therefore, the step creation energies relative to $\lambda_{\sigma}$ for $\mathrm{Ge}(100)$ have to be smaller than for $\mathrm{Si}(100)$. Since $\lambda_{S_{B}}$ of Ge determined below is almost an order of magnitude lower than on $\mathrm{Si}$, it seems to be justified to assume that the interaction forces on $\mathrm{Ge}(100)$ are generally smaller than on comparable $\mathrm{Si}(100)$ samples. Therefore, if we use the $\lambda_{d}$ parameter of $\mathrm{Si}(100)$, which is around $0.6 \mathrm{eV} / 2 a,{ }^{34}$ we get as upper estimate for $\lambda_{\sigma}$ for the $\mathrm{Ge}(100)-[011] 2.7^{\circ}$ surface $0.06 \mathrm{eV} / 2 a$.

$\lambda_{S_{B}}$ was determined from the diffusivity $\langle k\rangle^{2}$, which represents mainly the roughness of the $S_{B}$-type step. The kink creation energy $\epsilon_{S_{B}}$ derived from $\langle k\rangle^{2}$ is in the case of noninteracting kinks close to $\lambda_{S_{B}}$. Compared to $\mathrm{Si}(100), \lambda_{S_{B}}$ is five times lower. For a direct comparison with $\mathrm{Si}$, we assume that also $\lambda_{S_{A}}$ and $\lambda_{D_{B}}$ scale by this factor. Qualitatively, this can be rationalized by the fact that smooth $S_{A}$ and rough $S_{B}$ steps are also found on the $\mathrm{Ge}(100)$ surface, i.e., the energetic order of $\lambda_{S_{B}}>\lambda_{D_{B}}>\lambda_{S_{A}}$ is the same on Ge(100) as on $\mathrm{Si}(100)$.

With these assumptions, we plotted in Fig. 7 the critical terrace length calculated according to Eq. (4) as a function of $\lambda_{\sigma}$. The dashed-dotted line is for $\mathrm{Si}(100)$, where we used domain wall energies for $\mathrm{Si}(100)$ as calculated by Chadi, ${ }^{24}$ whereas the solid line corresponds to Ge. Due to the smaller difference of the step creation energies on vicinal Ge compared with $\mathrm{Si}$, a considerably higher step density for the transition to a doubly stepped surface is needed, in qualitative agreement with our findings.

Even semiquantitatively, our estimates are close to the experimental findings. With the estimate for $\lambda_{\sigma}$ given above, the $\mathrm{Ge}(100)-[011] 2.7^{\circ}$ surface clearly belongs to the regime with steps of single atomic height, whereas the $\mathrm{Ge}(100)-[011] 5.4^{\circ}$ surface is close to the transition line between a singly and doubly stepped surface. Experimen- 


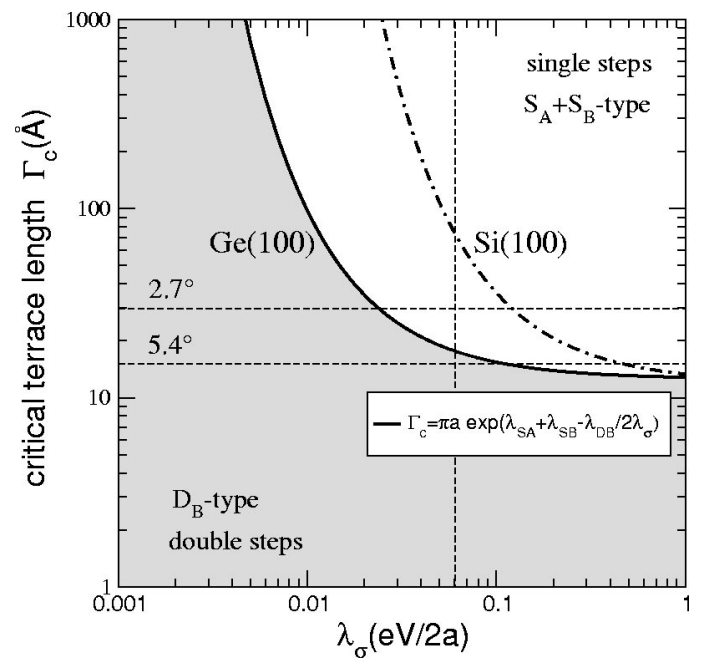

FIG. 7. Critical terrace length $\Gamma_{c}$ vs $\lambda_{\sigma}$. The dashed line was obtained by using the domain wall parameters for $\mathrm{Si}(100)$ calculated by Chadi. ${ }^{24}$ The solid line for $\mathrm{Ge}(100)$ is plotted with estimated parameters derived from our experiments. For details see text.

tally, the amount of $D_{B}$-type steps was found to be only around $50 \%$, with no indications for phase separation of singly and doubly stepped parts of the surface. This may be an indication that on vicinal Ge the transition between these two types of surfaces (both as a function of step concentration and of temperature) is continuous. A description of this behavior is not possible within the simple model used here.

\section{SUMMARY AND CONCLUSION}

We have shown in this paper how averages of microscopic properties of vicinal surfaces can be evaluated quantitatively by means of LEED. This requires the analysis of energy dependent spot splittings as well as of the twodimensional spot profiles both at Bragg positions and of the diffuse intensity. Since LEED is a priori an averaging method, all appropriate averages are already contained in the data.

Second, there are clear differences between $\mathrm{Si}$ and $\mathrm{Ge}$ in the surface morphology of vicinal surfaces, as demonstrated here. Steps of single atomic height turn out to be stable at much higher step concentrations on germanium. According to our analysis, this is mainly caused by the smaller difference between step creation energies of steps with single and double atomic height on Ge. Due to lower interaction forces between and along steps as compared to vicinal $\mathrm{Si}(100)$, $\mathrm{Ge}(100)$ surfaces are also more likely to show step structures with a high roughness. For further experiments concerning the epitaxial growth of $\mathrm{Si}$ on vicinal $\mathrm{Ge}(100)$ this circumstance can significantly influence the growth mode.

\section{ACKNOWLEDGMENT}

The financial support by the Deutsche Forschungsgemeinschaft and the $\mathrm{K}+\mathrm{S}$ Gruppe is gratefully acknowledged.
*Electronic address: pfnuer@fkp.uni-hannover.de

${ }^{\dagger}$ Present address: IBM Research Division, IBM T.J. Watson Research Center, Yorktown Heights, New York 10598.

${ }^{\ddagger}$ Present address: Institut für Laser- und Plasmaphysik, Universität Essen, Universitätsstraße 5, D-45117 Essen, Germany.

${ }^{1}$ N. C. Bartelt, J. L. Goldberg, T. L. Einstein, and E. D. Williams, Surf. Sci. 273, 252 (1992).

${ }^{2}$ H. J. W. Zandvliet and H. B. Elswijk, Phys. Rev. B 48, 14269 (1993).

${ }^{3}$ O. L. Alerhand, A. N. Berker, J. D. Joannopoulos, D. Vanderbilt, R. J. Hamers, and J. E. Demuth, Phys. Rev. Lett. 64, 2406 (1990).

${ }^{4}$ H. C. Jeong and E. D. Williams, Surf. Sci. Rep. 34, 171 (1999), see also references therein.

${ }^{5}$ X. Tong and P. A. Bennett, Phys. Rev. Lett. 67, 101 (1991).

${ }^{6} \mathrm{~J}$. W Gibbs, Collected Works (Longmans, London, 1928), Vol. 1.

${ }^{7}$ O. L. Alerhand, D. Vanderbilt, R. D. Meade, and J. D. Joannopoulos, Phys. Rev. Lett. 61, 1973 (1988).

${ }^{8}$ E. Pehlke and J. Tersoff, Phys. Rev. Lett. 67, 1290 (1991).

${ }^{9}$ Cf. with review paper from M. B. Webb, Surf. Sci. 299/300, 454 (1994).

${ }^{10}$ C. Schwennicke, J. Schimmelpfennig, and H. Pfnür, Surf. Sci. 293, 57 (1993).

${ }^{11}$ C. Tegenkamp, W. Ernst, M. Eichmann, and H. Pfnür, Surf. Sci. 466, 41 (2000).

${ }^{12}$ B. A. G. Kersten, L. Sjerps-Koomen, J. W. Zandvliet, and D. H. A. Blank, in Determining Nanoscale Physical Properties of Materials by Microscopy and Spectroscopy, edited by M. Sarikaya,
H. K. Wickramsinghe, and M. Issacson, Mater. Res. Soc. Symp. Proc. No. 332 (Materials Research Society, Pittsburgh, 1994), p. 555.

${ }^{13}$ X.-J. Zhang, G. Xue, A. Agarwal, R. Tsu, M.-A. Hasan, J. E. Greene, and A. Rockett, J. Vac. Sci. Technol. A 11, 2553 (1993).

${ }^{14}$ The scattering phase $S$, is defined as $S:=k_{\perp} d / 2 \pi, d=d_{\mathrm{Ge}}$ $=1.415 \AA, k_{\perp}=$ scattering vector of electrons perpendicular to the surface. $S=$ integer $n$ corresponds to a Bragg- or in-phase condition.

${ }^{15}$ S. D. Kevan, Phys. Rev. B 32, 2344 (1985).

${ }^{16}$ S. Fölsch, G. Meyer, K. H. Rieder, M. Horn-vonHoegen, T. Schmidt, and M. Henzler, Surf. Sci. 394, 60 (1997).

${ }^{17}$ B. A. G. Kersten, Ph.D. thesis, University of Twente, 1995.

${ }^{18}$ J. Wollschläger, F. Schäfer, and K. M. Schröder, Surf. Sci. 396, 94 (1998).

${ }^{19} \mathrm{C}$. Tegenkamp and J. Wollschläger (unpublished).

${ }^{20}$ Generally, in the case of adsorbate systems $G_{0}(\Theta)$ is written as $G_{0}(\Theta)=1-4 \Theta(1-\Theta)$. With the definition of $p$ (see text) and the definition of $\Theta:=\left\langle\Gamma_{i}\right\rangle\left\langle\left\langle\Gamma_{l}\right\rangle+\left\langle\Gamma_{s}\right\rangle[i=l\right.$ or $s$ with respect of the two solutions of $G_{0}(\Theta)$ ], it is easy to verify Eq. (2).

${ }^{21}$ C. Alfonso, J. M. Bermond, J. C. Heyraud, and J. J. Métois, Surf. Sci. 262, 371 (1992).

${ }^{22}$ J. Wollschläger and M. Larsson, Phys. Rev. B 57, 14937 (1998).

${ }^{23}$ D. E. Savage, J. Kleiner, N. Schimke, Y. H. Phand, J. Jacobs, R. Kariotois, and M. G. Lagally, J. Appl. Phys. 69, 1411 (1991).

${ }^{24}$ D. J. Chadi, Phys. Rev. Lett. 59, 1691 (1987).

${ }^{25}$ W. Ernst and H. Pfnür (unpublished).

${ }^{26}$ H. J. W. Zandvliet, H. B. Elswijk, E. J. van Loenen, and D. 
Dijkkamp, Phys. Rev. B 45, 5965 (1992).

${ }^{27}$ B. S. Swartzentruber, Y.-W. Mo, R. Kariotis, M. G. Lagally, and M. B. Webb, Phys. Rev. Lett. 65, 1913 (1990).

${ }^{28}$ H. J. W. Zandvliet, Phys. Rev. B 61, 9972 (2000).

${ }^{29}$ N. C. Bartelt, T. L. Einstein, and E. D. Williams, Surf. Sci. 276, 308 (1992).

${ }^{30}$ M. Giesen, Surf. Sci. 370, 55 (1997).
${ }^{31}$ N. C. Bartelt, T. L. Einstein, and E. D. Williams, Surf. Sci. Lett. 240, L591 (1990).

${ }^{32}$ T. W. Poon, S. Yip, P. S. Ho, and F. F. Abraham, Phys. Rev. Lett. 65, 2161 (1990).

${ }^{33}$ F. H. Stillinger and T. A. Weber, Phys. Rev. B 31, 5262 (1985).

${ }^{34}$ B. S. Swartzentruber, N. Kitamura, M. G. Lagally, and M. B. Webb, Phys. Rev. B 47, 13432 (1993). 